\title{
Comportamiento del Proceso Prehospitalario Ante la Llamada de Auxilio Por Trauma, CSC-911, 2011
}

\author{
Gabriel Sarmiento Ortiz, Vanessa Sarmiento León, Fray Martínez Reyes, Juan Carlos Salamea Molina
}

\section{RESUMEN}

Se reportó 4,665 llamadas reales que son el 5\% del total de llamadas de emergencia; $46 \%$ Trauma. $69,90 \%$ sexo masculino, relación 2,3:1. Grupo más afectado $20-29$ años 30,47\%. Traídos por el Cuerpo de Bomberos $72,71 \%$. Presencia de aliento etílico $6,42 \%$.Muertes 1,54\%. Accidente de tránsito 49,22\%; caídas $28,56 \%$; violencia $18,19 \%$; accidentes laborales $2,81 \%$ y quemaduras $1,22 \%$. Como politraumatismo $34,88 \%$, trauma de miembros $24,99 \%$, trauma de encéfalo-craneal $16,41 \%$, trauma maxilo-facial $12,40 \%$, trauma de columna $4,55 \%$, trauma torácico $4,55 \%$, trauma de abdomen $1,73 \%$ y trauma pélvico $0,75 \%$. Trauma contuso $73,14 \%$ y penetrante $26,86 \%$. Las instituciones públicas recibieron el $68,90 \%$. La media de transporte PH desde que sale fue 11'55"; medina 05'; moda 04'. Tiempo desde la llamada hasta la llegada al hospital la media fue 17'30"; media 9', moda 8'. El tiempo de respuesta de la unidad intra-institucional es 7'06"; media 3'; moda 2'. Los resultados obtenidos concuerdan con la accidentalidad de una ciudad desarrollada. Existe un uso inadecuado del sistema 911 lo que retarda el servicio de las unidades e emergencia. El cuerpo de bomberos juega un papel fundamental en atención $\mathrm{PH}$ por trauma. Este trabajo será determinante para tomar directrices para mejorar la atención $\mathrm{PH}$ del trauma.

Palabras claves: Trauma, 911, atención prehospitalaria.

\begin{abstract}
4,665 real emergency calls, that represent $5 \%$ of the total emergency calls; $46 \%$ were due trauma. $69.9 \%$ of the calls were from males, with a 2.3:1 relation. Most affected group between 20 and 29 years. Brought by the fire brigade $72.71 \%$. Ethyl breath found in $6.42 \%$. Deaths: $1.54 \%$. Traffic accidents: $49.22 \%$; falls $28.56 \%$; violence $18.19 \%$; work accidents $2.81 \%$ and burns $1.22 \%$. As polytrauma $34.88 \%$, member trauma $24.99 \%$, traumatic brain injury $16.41 \%$, maxilla-facial trauma $12.4 \%$, spinal column trauma $4.55 \%$, thoracic trauma $4.55 \%$, abdominal trauma $1.73 \%$ and pelvic trauma $0.75 \%$. Blunt trauma $73.14 \%$ and penetrating trauma $26.86 \%$. Public Health institutions received $68.9 \%$. Prehospital transport mean since the departure was 11'55', median 05', and mode 04'. Time passed since call until hospital arrival: mean 17'30", media 9', mode 8'. Answer time in the institutional unit was 7'06" (mean), 3' (median), 2' (mode). The obtained results are in agreement with the accidentally rate of a developed city. There's an inadequate use of the 911 system, which delays the emergency's unit services. The fire brigade plays a fundamental part in prehospital attention to trauma. This study would be fundamental in order to improve the directions in $\mathrm{PH}$ attention to trauma.
\end{abstract}

Keywords: Trauma, 911, Emergency, Prehospital attention.

How to cite this article: Ortiz GS, León VS, Reyes FM, Molina JCS. Comportamiento del Proceso Prehospitalario Ante la Llamada de Auxilio Por Trauma, CSC-911, 2011. Panam J Trauma Critical Care Emerg Surg 2012;1(3):168-174.

Source of support: Nil

Conflict of interest: None declared

\section{INTRODUCCIÓN}

El Trauma se ha convertido en una de las principales causas de muerte y discapacidad. Las lesiones producidas son ya un problema de salud pública importante en todo el mundo. Cada día, 16,000 personas mueren a causa de trauma, y por cada persona que muere, hay varios miles de heridos más, muchos de ellos con secuelas permanentes. Representa el 16\% de la carga mundial de morbilidad. La carga de mortalidad y discapacidad por lesiones es especialmente notable en países de bajos y medianos ingresos; aproximadamente el 90\%, se produce en estos países. ${ }^{1-3}$

La Ciudad de Cuenca con un área de $120 \mathrm{Km}^{2}$, queda ubicada en la Provincia del Azuay de la republica del Ecuador, cuenta con 505,585 habitantes según el censo de población y vivienda realizado en el 2010 (INEC) 4 con 53\% mujeres y $47 \%$ hombres.

El trauma es uno de los principales problemas que afectan la salud de la población en el Ecuador; convirtiéndose en la primera causa de mortalidad con $16,8 \%$ estando sobre las enfermedades metabólicas, respiratorias agudas y cardiovasculares según datos del 2007.

La falta de jerarquización del trauma como enfermedad y epidemia, no permite una respuesta gubernamental con programas de atención y prevención, sumados a la dificultad para generar un registro adecuado y diagnóstico de la situación real, los problemas que pueden generar esto son: - Ausencia de datos epidemiológicos locales sobre los traumatismos

- La diferencia entre los datos provenientes de distintas fuentes, incluso de los pocos reportes locales que existen.

- La falta de compromiso por parte del personal de salud para evitar el mal registro o subregistro de datos. ${ }^{5}$

Todo esto no permite una visión real de la problemática entorno al trauma y la atención que esta merece a todos los niveles tanto primario, secundario y terciario.

Hasta el primer trimestre del 2012 el Consejo de Seguridad Ciudadana (CSC) estuvo a cargo de la central 9-1$1^{6}$, en la actualidad el gobierno central generó un programa con el nombre 'Mi Emergencia', el mismo que está dirigido a la atención del trauma y emergencias en varias fases entre ellas el desarrollo de un sistema pre-hospitalario con el nombre de 'SIS-ECU 911' Institución que a nivel nacional esta respondiendo a las llamadas de emergencia. Este trabajo se convierte en un hito para el mejor desarrollo de estos programas y para futuras evaluaciones y comparaciones.

Paper presented at the student competition, 25th Annual Congress of Panamerican Trauma Society, Medellin, Colombia, November 2012. 
La información recogida ha servido para mostrar una estadística general, sin embargo, hace falta una sistematización para que la misma pueda ser utilizada en pro de medidas preventivas y de promoción de la salud.

Al ser el trauma una línea de trabajo dinámica y permanente, es necesario aprovechar la oportunidad para desarrollar investigación útil, dentro y fuera de los espacios universitarios.

\section{OBJETIVO GENERAL}

Descripción de las características del trauma y su valoración pre-hospitalaria en la ciudad de Cuenca, y su distribución, según los reportes de llamadas de emergencia 'Consejo de Participación Ciudadana 9-1-1.'

\section{OBJETIVOS ESPECÍFICOS}

- Identificar las zonas geográficas de la Ciudad de Cuenca de acuerdo a la incidencia de los eventos de trauma.

- Establecer los lugares y su relación de accidentalidad en los que se produce el trauma de acuerdo a la ubicación de las víctimas.

- Evaluar el tiempo de llegada del servicio de atención pre-hospitalaria.

- Identificar las características de la ocurrencia del trauma de acuerdo a las variables estudiadas.

- Identificar la relación que existe entre el trauma y el aliento etílico.

\section{MATERIALES Y MÉTODOS}

Es un estudio retrospectivo de la base de datos del CSC. Comprende el período de 1 año, desde el 1 de enero al 31 de diciembre del 2011. Se ubicó a cada una de las emergencias en el mapa de la ciudad de Cuenca según el sector (parroquia) correspondiente y se procedió recolectar esta información en un instrumento construido para el efecto (Anexo 1). Los criterios de inclusión fueron todas aquellas llamadas reales de emergencias que estaban en relación a eventos traumáticos (lesión severa a nivel orgánico, resultante de la exposición aguda a un tipo de energía mecánica, térmica, eléctrica, química o radiante, en cantidades que exceden el umbral de la tolerancia fisiológica), y que contaban con la información completa requerida para el anexo 1 . Se excluyeron los registros con información incompleta.

Una vez recolectada la información se procedió a trasladarla a una base de datos construida en el software SPSS V 19,0; se realizaron tablas y gráficos según sea el caso, utilizándose estadística descriptiva para la misma. Para las variables cualitativas se utilizaron tablas de frecuencias; las cuantitativas se analizaron con medidas de tendencia central y de dispersión. Fue necesario exportar algunos datos al programa Excel 2009 para su procesamiento.

La información recolectada fue: fecha en relación al año mes y día, edad, sexo, lugar donde sucedió el trauma según la parroquia a la que pertenece, hora de la llamada, hora de salida de la Institución, hora de salida de la unidad de rescate con respecto a la llamada de auxilio de rescate y hallazgos. Presencia de aliento etílico. Etiología del trauma: Accidente de tránsito, quemadura, violencia, accidente laboral, caídas. Trauma: Contuso o penetrante según el reporte del operador. Área corporal: según descripción del operador: cabeza, tórax, abdomen, pelvis, columna vertebral, miembros y poli traumatizado. Tipo de energía: cinética, térmica, eléctrica, química y nuclear. : Intuición de salud a la que fue trasladada la emergencia del trauma. Institución de Asistencia: Bomberos, Cruz rojo, Policía, Defensa civil, ninguna. Mortalidad: vivo o muerto.

Anexo 1: Registro de la base de datos

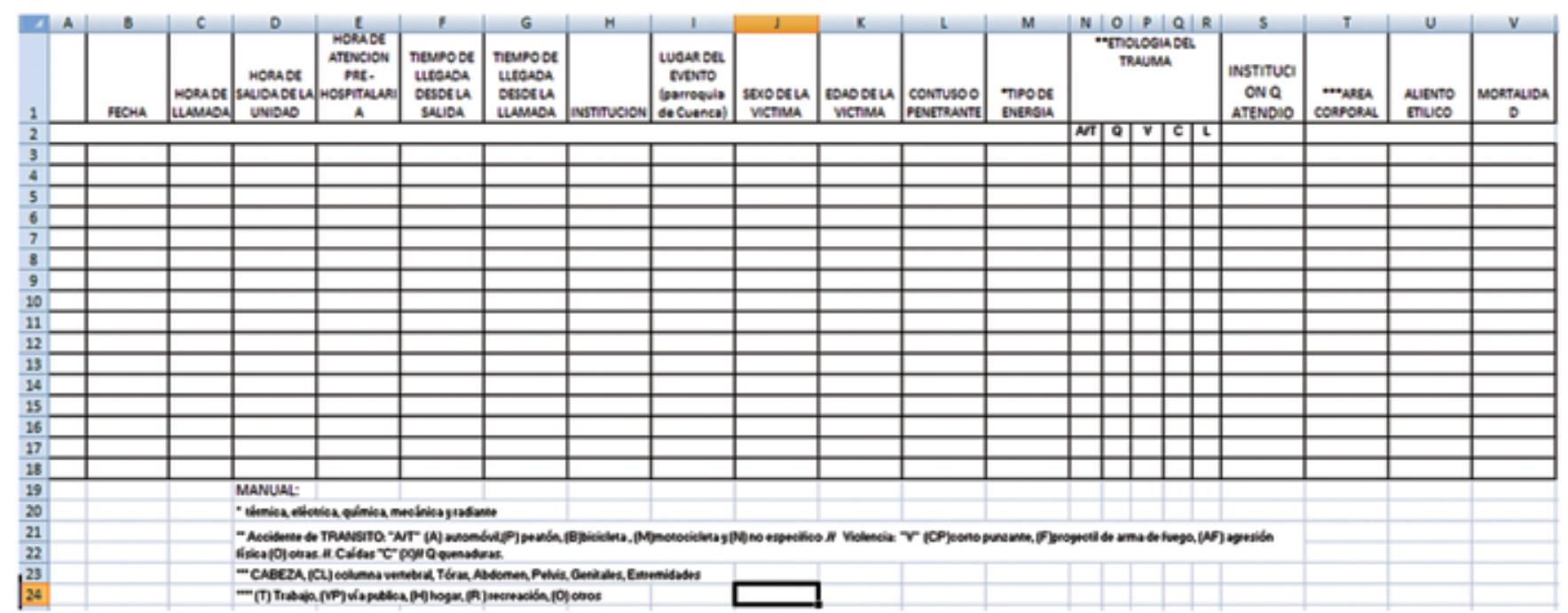




\section{RESULTADOS}

Se registraron 4,665 llamadas que corresponden al 5\% del total de llamadas reales al CSC. Encontramos 2,122 pacientes calificados como eventos traumáticos, correspondiendo al $46 \%$. La distribución según la edad puede verse en la Grafico 1, la mayor distribución está en el grupo de 20-29 años con una frecuencia del $30,5 \%(n=644)$, seguido por el de $10-19$ y $30-39$, con lo que el grupo etario de 10 a 39 años hace un porcentaje acumulado del $65 \%$, siendo el grupo más afectado y vulnerable.

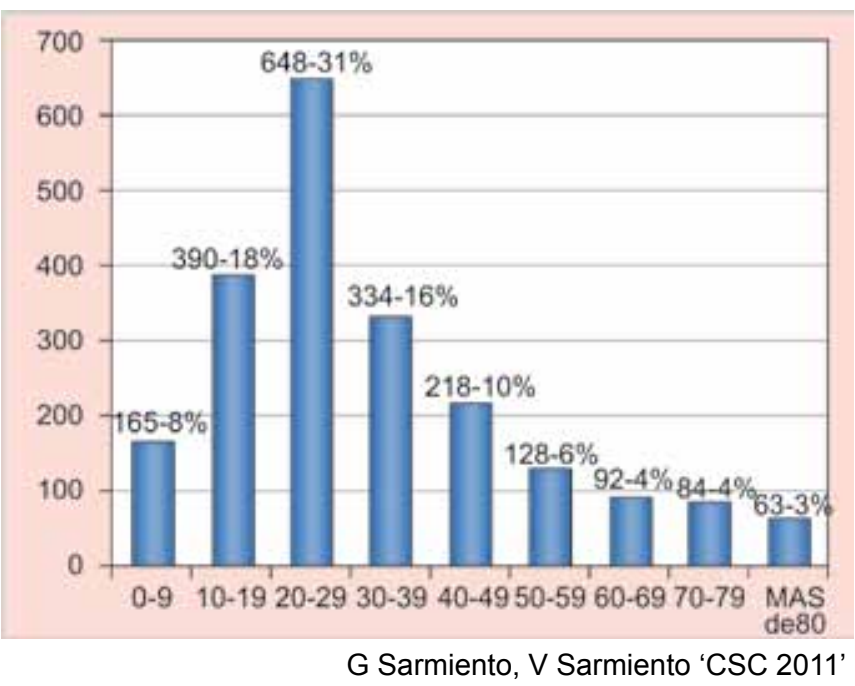

Gráfico 1: Distribución del trauma por edad
Gráfico 2, muestra que el sexo masculino constituye el $70 \%$ versus el $30 \%$ del sexo femenino, con una relación de $2,3: 1$

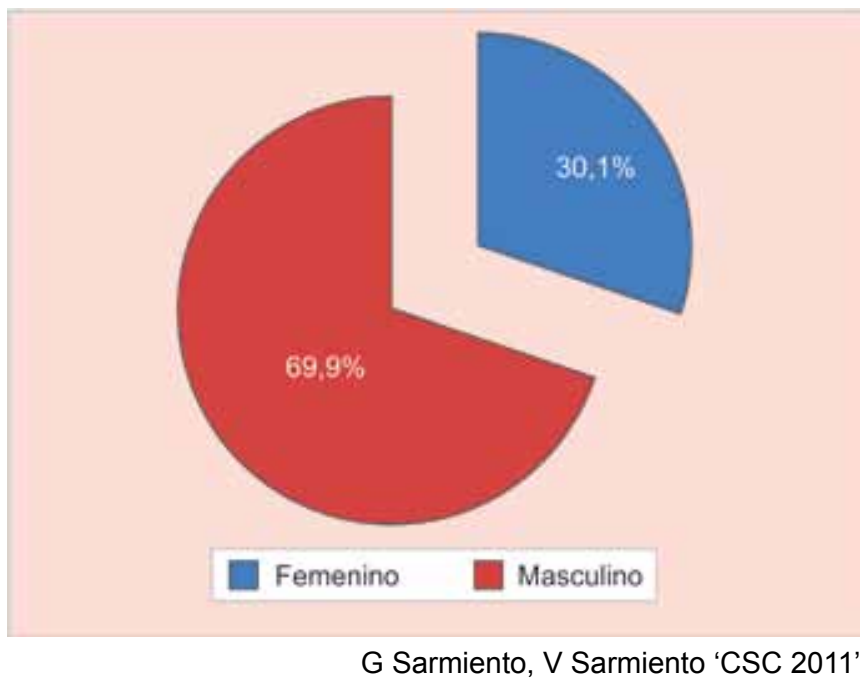

Gráfico 2: Trauma por sexo

En la Tabla 1 y Gráfico 3, se evidencia que la incidencia del trauma en el área urbana es de $84 \%$, en comparación con el $16 \%$ del área rural.

La incidencia del trauma en las diferentes parroquias urbanas de cuenca se describe en el Gráfico 4, en donde observamos que Yanuncay, San Sebastián, Totoracocha, El Vecino y El Batán suman el 50\% del trauma, y en las 10 parroquias restantes el otro $50 \%$.

\begin{tabular}{|c|c|c|c|c|c|c|c|}
\hline \multicolumn{4}{|c|}{ Urbano } & \multicolumn{4}{|c|}{ Rural } \\
\hline Parroquia & $n$ & Urbano (\%) & Total & Parroquia & $n$ & Rural & Total \\
\hline Bellavista & 140 & 7,86 & 6,60 & Baños & 46 & 13,49 & 2,17 \\
\hline Cañaribamba & 61 & 3,43 & 2,87 & Checa & 2 & 0,59 & 0,09 \\
\hline El Batan & 155 & 8,70 & 7,30 & Chiquintad & 1 & 0,29 & 0,05 \\
\hline EI Sagrario & 125 & 7,02 & 5,89 & Cumbe & 25 & 7,33 & 1,18 \\
\hline El Vecino & 165 & 9,26 & 7,78 & EI Valle & 46 & 13,49 & 2,17 \\
\hline GR Davalos & 88 & 4,94 & 4,15 & Molleturo & 44 & 12,90 & 2,07 \\
\hline Hno Miguel & 48 & 2,70 & 2,26 & Nulti & 38 & 11,14 & 1,79 \\
\hline Huaynacapac & 120 & 6,74 & 5,66 & O Cordero & 1 & 0,29 & 0,05 \\
\hline Machangara & 59 & 3,31 & 2,78 & Paccha & 16 & 4,69 & 0,75 \\
\hline Monay & 81 & 4,55 & 3,82 & Quingeo & 8 & 2,35 & 0,38 \\
\hline San Blas & 94 & 5,28 & 4,43 & Ricaurte & 35 & 10,26 & 1,65 \\
\hline S Sebastian & 167 & 9,38 & 7,87 & S Joaquin & 9 & 2,64 & 0,42 \\
\hline Sucre & 106 & 5,95 & 5,00 & Santa Ana & 6 & 1,76 & 0,28 \\
\hline Totoracocha & 166 & 9,32 & 7,82 & Sidcay & 2 & 0,59 & 0,09 \\
\hline \multirow[t]{4}{*}{ Yanuncay } & 206 & 11,57 & 9,71 & Sinincay & 12 & 3,52 & 0,57 \\
\hline & & & & Tarqui & 26 & 7,62 & 1,23 \\
\hline & & & & Turi & 18 & 5,28 & 0,85 \\
\hline & & & & Portete & 6 & 1,76 & 0,28 \\
\hline Total & 1781 & $100 \%$ & 83,93 & & 341 & 100 & 16,07 \\
\hline
\end{tabular}

G Sarmiento, V Sarmiento 'CSC 2011' 


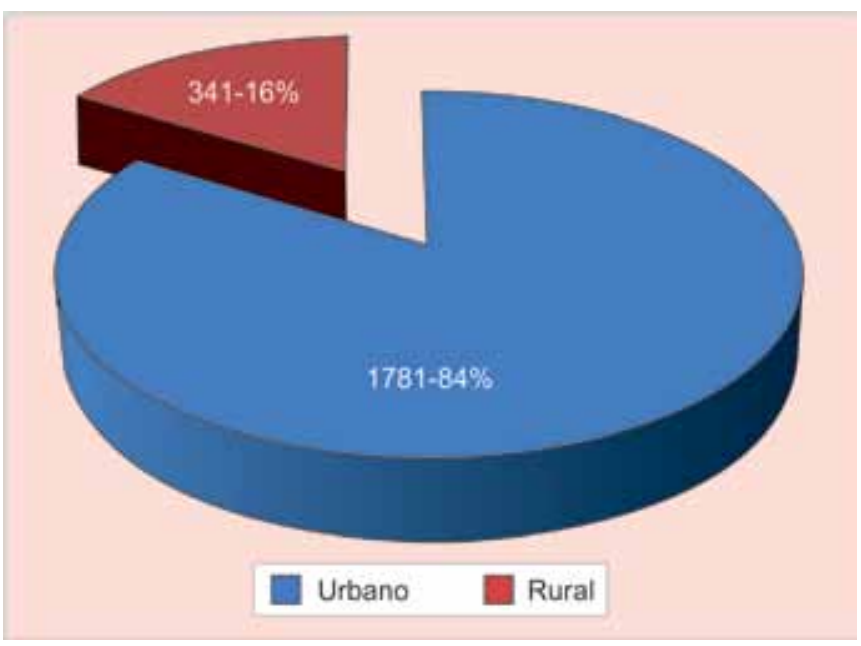

G Sarmiento, V Sarmiento 'CSC 2011'

Gráfico 3: Distribucion del trauma de acuerdo al area urbana-rural

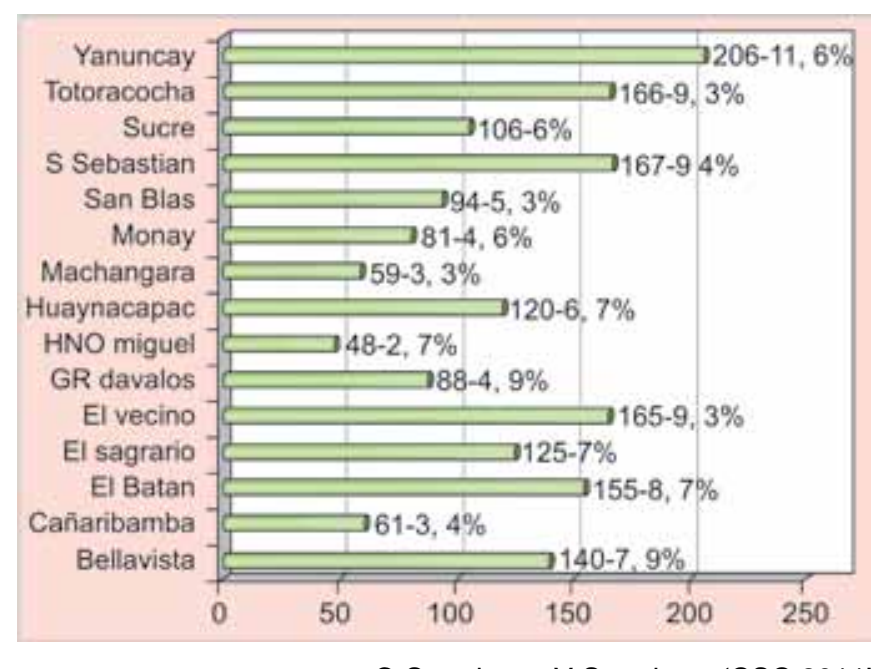

G Sarmiento, V Sarmiento 'CSC 2011'

Gráfico 4: Trauma en las parroquias urbanas de Cuenca

Con respecto a las instituciones de auxilio que atendió el trauma notamos en el Gráfico 5 que el Cuerpo de Bomberos de Cuenca, acudió al 72\%, la Cruz roja al 27,10\%; Policía 0,66\%; y Defensa Civil 1 caso.

La institución de salud pública "Hospital Vicente Corral Moscoso" atendió el 54\%; el hospital de la seguridad social "IESS" el 15\%; el 14\% atención pre-hospitalaria, y el 17\% en instituciones privadas, como se muestran en el Gráfico 6 .

El en Gráfico 7 observamos las distribución del trauma según las parroquias urbanas, señalando de color rojo las más afectadas (más de 200 casos); anaranjado (150-199); amarillo (100-149); verde olivo (50-99) y verde claro (menos de 50).

En el Gráfico 8 la ubicación de las diferentes instituciones de servicio de emergencia y de salud a las que acude el trauma.

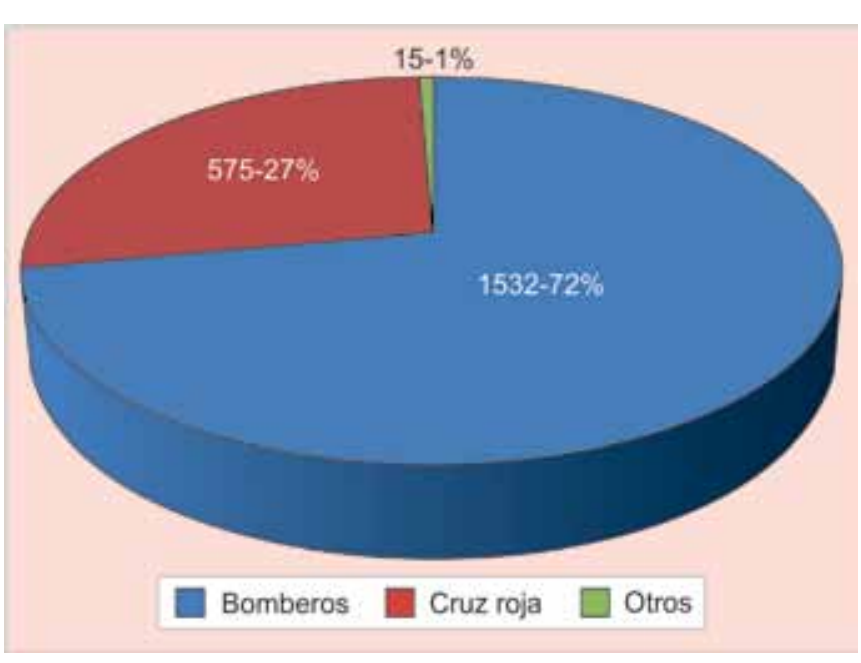

G Sarmiento, V Sarmiento 'CSC 2011'

Gráfico 5: Institucion de auxilio que atendio el trauma

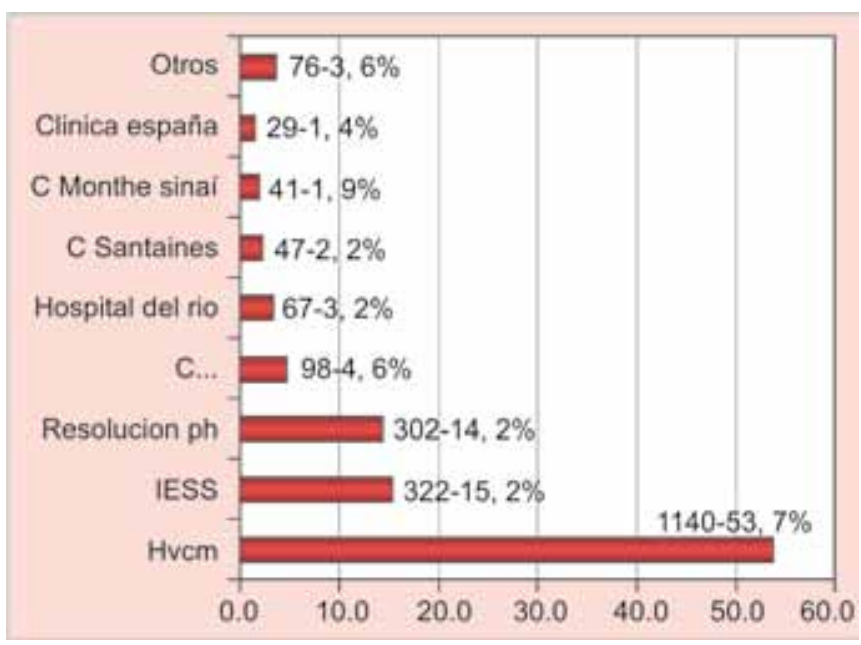

G Sarmiento, V Sarmiento 'CSC 2011'

Gráfico 6: Institucion de salud que recibió el trauma

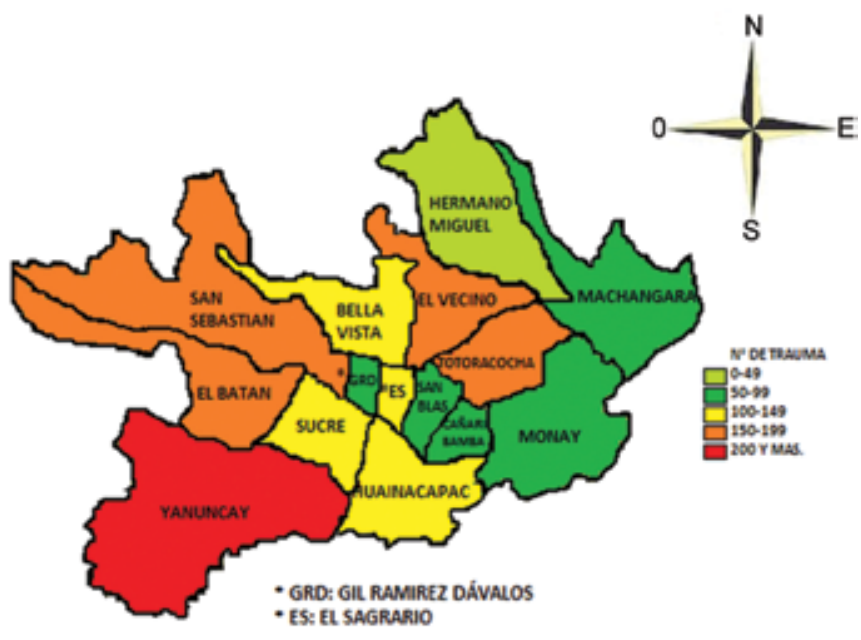

G Sarmiento, V Sarmiento 'CSC 2011'

Gráfico 7: Distribucion del trauma en las parroquias urbanas de cuenca 
En los Gráficos 9, 10 y 11 se indica la etiología del trauma urbano, de colores rojo, anaranjado, amarillo y verde, de mayor a menor frecuencia, respectivamente.

El Gráfico 12, representa la distribución del trauma y su intensidad en las parroquias rurales.

El Gráfico 13 indica que mayoritariamente el trauma ocurre entre las 12:01 y 18:00 horas 34\%, no así de 00:01 a 06:00 que es el 13\%.

La media de transporte $\mathrm{PH}$ desde que se despacha la unidad de rescate fue 11'55' (medina 05'; moda 04'). El tiempo desde la recepción de la llamada hasta la llegada a la institución de salud la media fue 17'30"' (media 9', moda 8'). El tiempo de respuesta de la unidad intrainstitucional es de una media de 7'06' (media 3'; moda 2').

En esta serie se encontró el registro de la presencia de aliento etílico en $6,42 \%$.

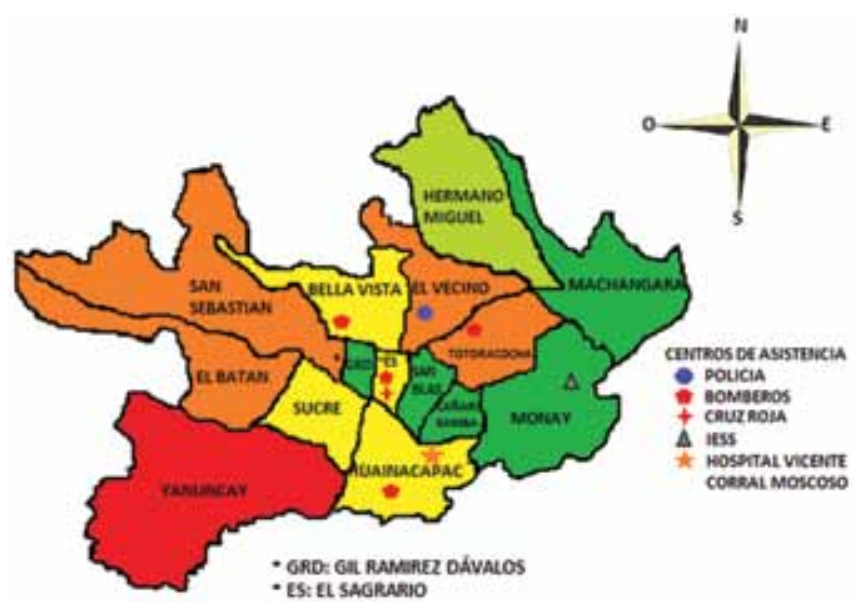

G Sarmiento, V Sarmiento 'CSC 2011'

Gráfico 8: Distribucion de las instituciones de emergencia y su relacion con el trauma

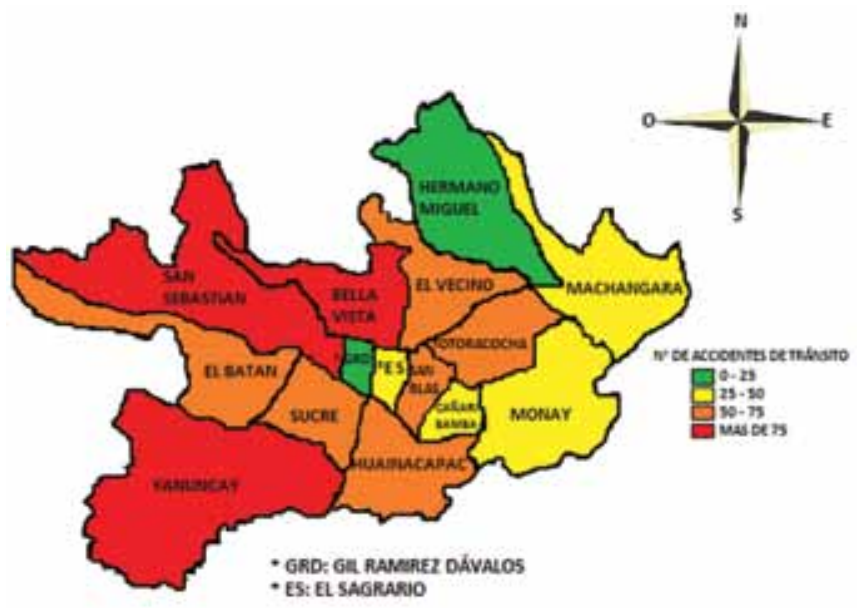

G Sarmiento, V Sarmiento 'CSC 2011'

Gráfico 9: Distribucicion de los accidentes de transito y su realcion con el trauma en la ciudad de cuenca, area urbana

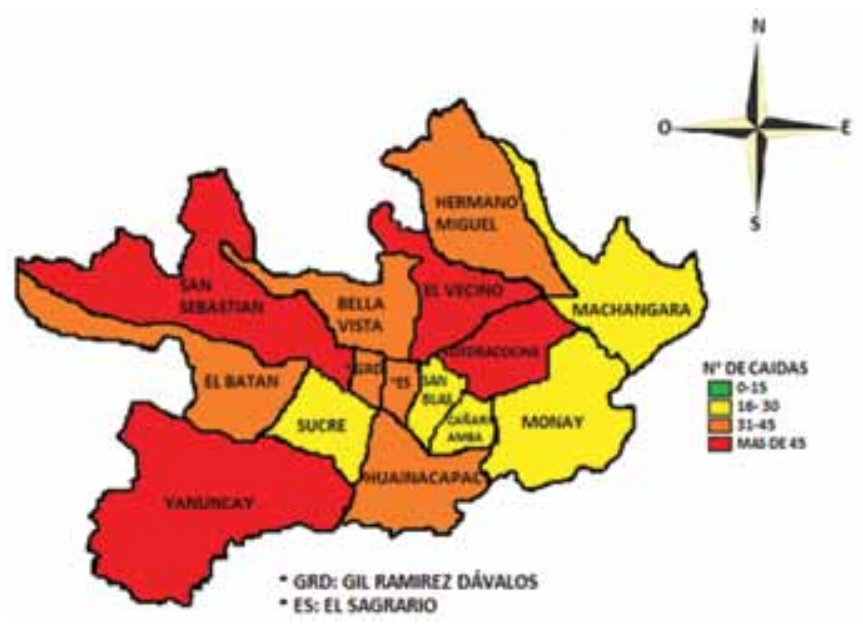

G Sarmiento, V Sarmiento 'CSC 2011'

Gráfico 10: Distribucion de las caidas y su relacion con el trauma en la ciudad de cuenca, area urbana

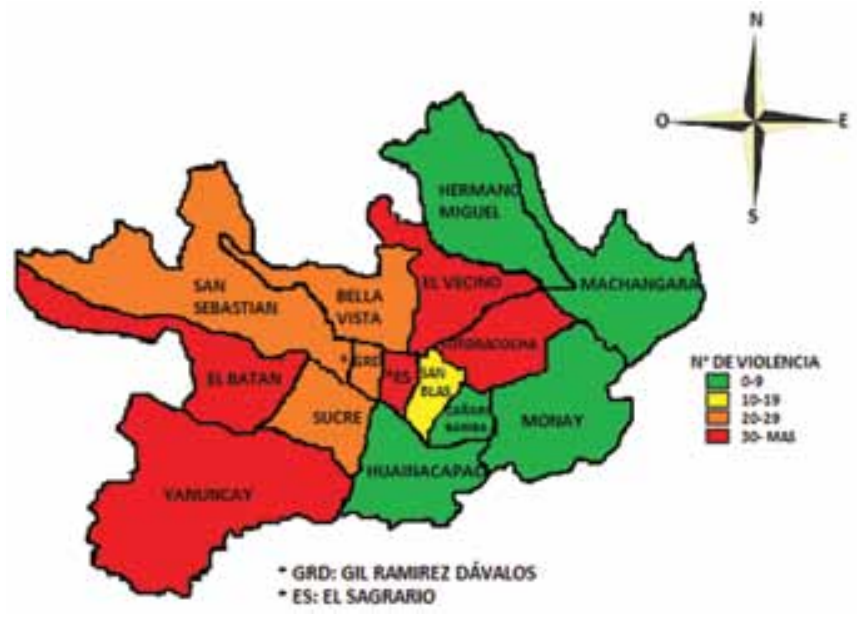

G Sarmiento, V Sarmiento 'CSC 2011'

Gráfico 11: Distribucion de la violencia y su relacion con el trauma en la ciudad de cuenca, area urbana

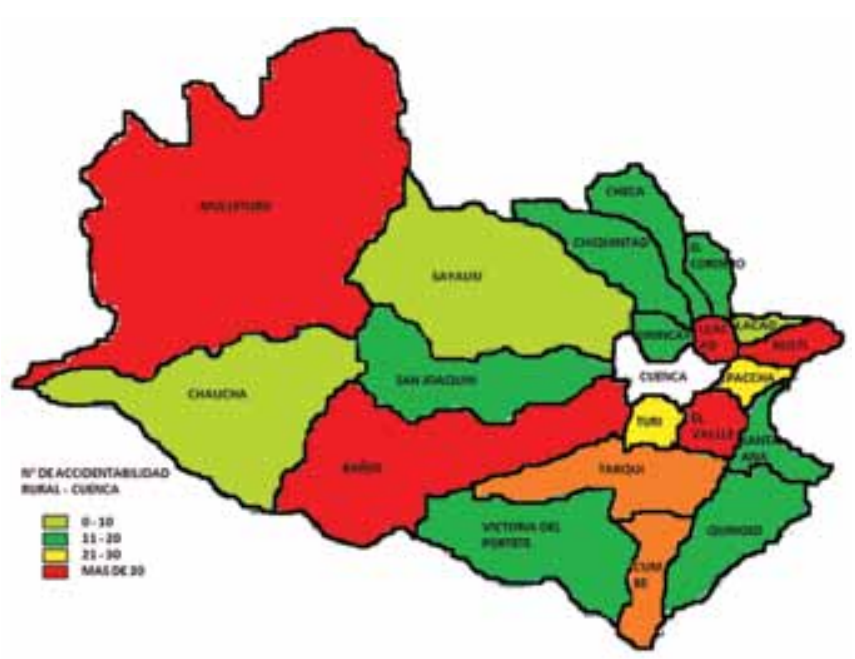

G Sarmiento, V Sarmiento 'CSC 2011'

Gráfico 12: Distribucion rural del trauma de la ciudad de cueca 


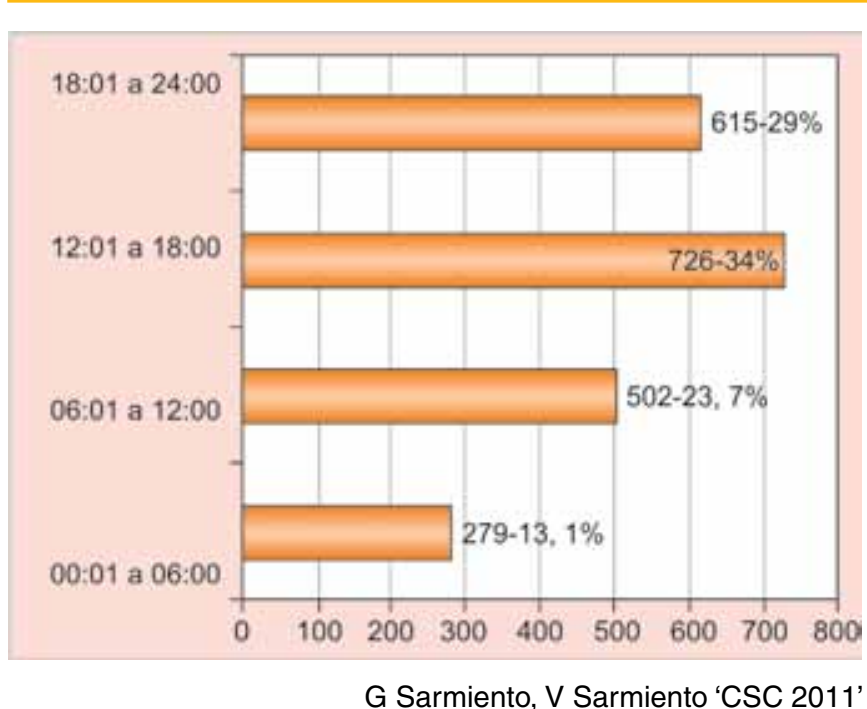

Gráfico 13: Trauma en las 24 horas del dia

Análisis El estudio realizado en la Cuidad de Cuenca nos muestra que existe mayor trauma en el sexo masculino $70 \%$ con respecto al sexo femenino $30 \%$, con mayor predominio personas económicamente activas 31\%(20-29 años), disminuyendo a partir de los 50 años $17 \%$; lo que concuerda con la literatura revisada.

La distribución del trauma nos indica que la zona oeste que comprende las parroquias de Yanuncay, San Sebastián y el Batan corresponden al $29,64 \%$ de las llamadas de emergencia urbana y $24,88 \%$ del total de las llamadas por trauma en la ciudad siendo esta la zona donde se atendieron la mayorías de emergencias; las parroquias del Norte, Bellavista, El vecino y Hermano Miguel corresponde al $19,82 \%$ urbanas y $16,63 \%$ del total de pacientes con trauma; en el centro Gil Ramírez Dávalos, El Sagrario y San Blas el $17,23 \%$ urbana y del total de llamadas $14,46 \%$; la zona del este ciudad Machangara, Totoracocha, Cañaribamba y Monay se atendieron el 20,60\% urbana y el 17,29\% del total; por último en el Sur Huayna-Capac y Sucre tuvieron el $12,68 \%$ urbana y $10,65 \%$ del total.

Las instituciones públicas reciben la mayor parte de emergencias diagnosticadas como trauma 69\%, distribuidas $54 \%$ en el hospital público 'Vicente Corral Moscoso', y $15 \%$ en el hospital 'José Carrasco Arteaga' que pertenece al instituto se Seguridad Social; mientras que el 14\% recibe atención pre-hospitalaria y el 17\% en instituciones privadas.

El cuerpo de Bomberos es la institución de rescate que mas atiende las emergencias por trauma en un $73 \%$, se cree que esto se debe a que esta institución tiene 4 estaciones de servicio en diferentes sectores de la ciudad, no así la Cruz Roja que tiene una sola estación localizada en el centro de Cuenca.

Las llamadas de auxilio se receptan las 24 horas del día, siendo mayor la accidentabilidad entre las entre las 12:01 y $24: 00$ horas $63 \%$ y el $37 \%$ entre las $00: 01$ y $12: 00$ horas.
$\mathrm{Al}$ revisar los tiempos de respuesta vemos un adecuado despacho de las unidades de auxilio, se registra un tiempo mayor de respuesta intra-institucional, secundario a la gran cantidad de llamadas falsas de auxilio, que se registran.

No se registra ninguna relación entre frecuencia y los eventos traumáticos, esto se debe a la subjetividad de este hecho, la falta de objetividad y el subregitro debido a las implicaciones legales.

\section{CONCLUSIONES}

El trauma es la principal causa de emergencias en la ciudad de Cuenca ya que el $46 \%$ de las llamadas al CSC fueron por trauma, afecta principalmente al sexo masculino, con una relación de 2,3:1 sobre el femenino. Con mayor incidencia entre los 20 a 29 años de edad 30\%. La principal causa de trauma son los accidentes de tránsito con $49,22 \%$, y como su consecuencia principal los politraumatismos $34,8 \%$, luego el trauma de miembros el $25 \%$ y en tercer lugar el trauma de cráneo en un $16,4 \%$. La diferencia entre el área urbana y rural es bastante significativa del $84 \%$ en el área urbana en una relación a la rural de 5,3:1. Las Instituciones de Salud Publica y Seguridad Social tienen gran importancia en la atención del trauma ya que reciben el $69 \%$ de los traslados de los pacientes afectados, cabe recalcar que la resolución pre-hospitalaria es de un $15 \%$ y el resto de pacientes con trauma son atendidas por instituciones privadas de la ciudad. El cuerpo de Bomberos atiende el $73 \%$ del trauma, seguido de la Cruz Roja 27\%. Notamos que las zonas de mayor riego son la Parroquia de Yanuncay. Según la etiología el trauma ocurre en las personas en edad económicamente activa, las caídas afectan mayormente a las personas mayores de 65 años. No existe relación entre el aliento etílico y la etiología del trauma, lo que no concuerda con estudios revisados, lo que nos hace pensar en un posible sub-registro por parte de la persona que atendió el trauma, ya que solo el 6,4\% reporto aliento etílico. La mortalidad en relación al trauma mostro un índice del 1,54\%; predominando la violencia como causante del mismo.

\section{RECOMENDACIONES}

1. El SIS-ECU 911, debe tomar en cuenta estos resultados para plantearse estrategias de atención pre-hospitalaria del trauma.

2. Replantear la ubicación de los servicios de emergencia según el mapeo del trauma, además se pueden plantear estrategias dirigidas especialmente hacia el tránsito y violencia.

3. Las caídas corresponden a la segunda etiología de los eventos traumáticos y es la primera en el grupo del adulto 
mayor, registrándose con mayor frecuencia en la zona central de la cuidad (centro histórico), lo que demuestra que la ciudad no es apta para el peatón.

4. Sistematizar e implementar un registro informático con el fin de evitar los subregistros.

\section{REFERENCIAS}

1. Joshipura M. Guidelines for essential trauma care [Internet]. 2006. Available from: http://www.ncbi.nlm.nih.gov/pubmed/20661563

2. LDEM, R ANAMK. Consideraciones epidemiológicas sobre los traumatismos en Chile * An epidemiological review of accidents and violence in Chile. 2007;59.

3. Greaves I, Porter K, Ryan JM. Trauma care manual [Internet]. Arnold; 2000 [cited 2012 Jan 29]. Available from: http:// onlinelibrary.wiley.com/doi/10.1002/cbdv.200490137/abstract

4. INEC, Instituto Ecuatoriano de Estadísticas y Censo. Estadísticas de la Semana. 2010. 2010;:1.

5. Astudillo R, Salamea JC, Crespo P, Salamea P. Trauma, Diez Años De Experiencia, Hospital Vicente Corral Moscoso. Revista Trauma de la Sociedad Ecuatoriana de Trauma. 2007;1(1):2-3.
6. CSC Consejo de Seguridad Ciudadana Cuenca [Internet]. 2012;:1. Available from: http://www.csc.gob.ec/CSC/

\section{ACERCA DE LOS AUTORES}

\section{Gabriel Sarmiento Ortiz}

Estudiante de la Facultad de Medicina, Universidad del Azuay

\section{Vanessa Sarmiento León}

Estudiante de la Facultad de Medicina, Universidad del Azuay

\section{Fray Martínez Reyes}

Profesor de la Facultad de Medicina, Universidad del Azuay

\section{Juan Carlos Salamea Molina}

(Correspondiente Autor)

Profesor de la Facultad de Medicina, Universidad del Azuay e-mail: jsalamea@gmail.com 